\title{
Squamous morules are functionally inert elements of premalignant endometrial neoplasia
}

\author{
Ming-Chieh $\operatorname{Lin}^{1,2, *}$, Lesley Lomo ${ }^{1, *}$, Jan PA Baak ${ }^{3}$, Charis Eng ${ }^{4,5}$, Tan A Ince ${ }^{1}$, \\ Christopher P Crum ${ }^{1}$ and George L Mutter ${ }^{1}$
}

${ }^{1}$ Department of Pathology, Brigham and Women's Hospital, Boston, MA, USA; ${ }^{2}$ Department of Pathology, National Taiwan University Hospital and National Taiwan University College of Medicine, Taipei, Taiwan; ${ }^{3}$ Department of Pathology, Stavanger University Hospital, Stavanger, Norway and The Grade Institute, University of Bergen, Bergen, Norway; ${ }^{4}$ Genomic Medicine Institute and Taussig Cancer Center, Cleveland Clinic Foundation, Cleveland, OH, USA and ${ }^{5}$ Department of Genetics and CASE Comprehensive Cancer Center, Case Western Reserve University School of Medicine, Cleveland, OH, USA

\begin{abstract}
Squamous morules are a common component of premalignant glandular lesions that are followed by glandular, rather than squamous, carcinomas. We tested the hypothesis that the appearance of glands associated with morules predicts cancer risk, and undertook molecular testing to determine the clonal and hormonal response properties of admixed squamous and glandular elements. A total of 66 patients with squamous morules in an index endometrial biopsy had follow-up clinical data (average follow-up: interval 31 months, 2.5 biopsies) showing development of carcinoma in $11 \%(7 / 66)$ of cases. The histological appearance of morule-associated glands in the index biopsy was significantly associated with this clinical outcome, with the majority $(71 \%, 5 / 7)$ of cancer occurrences following an overtly premalignant lesion (endometrial intraepithelial neoplasia) with squamous morules. Eight endometrial intraepithelial neoplasias with squamous morules were examined by immunohistochemistry for estrogen and progesterone receptors and mitotic activity (Ki-67 antigen percent stained). Glandular components had abundant estrogen and progesterone receptors, and high levels of mitotic activity in all cases. In sharp contrast, all squamous morules were devoid of sex hormone receptors and had undetectable or extremely low-proliferation rates. When mutated, the same specific PTEN mutation was detected in squamous and glandular elements, indicating that both are of common lineage. The clinical and laboratory data are consistent with a model of morule biology in which squamous morules are a hormonally incompetent subpopulation of endometrial glandular lesions. Isolated morules might result from artifactual displacement from their native glandular context, or selective hormonally induced regression of the glandular but not squamous components over time. Subsequent cancer risk, as promoted by estrogens, is greatest when the glandular component has the appearance of endometrial intraepithelial neoplasia. Even isolated morules should be carefully followed, however, to exclude a coexisting undersampled, or occult, glandular lesion.
\end{abstract}

Modern Pathology (2009) 22, 167-174; doi:10.1038/modpathol.2008.146; published online 19 September 2008

Keywords: endometrial hyperplasia; endometrial intraepithelial neoplasia; metaplasia; sex hormones; squamous morules

Endometrial adenocarcinomas are readily classified into 'endometrioid (type I)' and 'non-endometrioid (type II)' subsets based on differing clinicopatholo-

Correspondence: Dr GL Mutter, MD, Division of Women's and Perinatal Pathology, Department of Pathology, Brigham and Women's Hospital, 75 Francis Street, Boston, MA 02115, USA.

E-mail: gmutter@rics.bwh.harvard.edu

*Theses authors contributed equally to this work.

Received 16 June 2008; revised 6 August 2008; accepted 7 August 2008; published online 19 September 2008 gic and molecular features. ${ }^{1}$ Endometrioid endometrial cancers, comprising over $90 \%$ of all endometrial glandular neoplasms, are distinguished by inactivation of the PTEN tumor suppressor pathway and a prodrome of long-term estrogen exposure unopposed by progestins. The indolent beginnings of endometrioid endometrial adenocarcinomas may be manifest by emergence of a premalignant glandular lesion (endometrial intraepithelial neoplasia (EIN) or atypical endometrial hyperplasia), ${ }^{2}$ several years in advance. The non-endometrioid class, 
exemplified by prototypical papillary serous histomorphology, is not associated with estrogen exposure and is characterized by inactivation of the p53 pathway and very aggressive clinical behavior.

Heterotopic differentiation or 'metaplasia,' is an altered differentiated cellular phenotype seen as a predominant or minor pattern of some type I, or endometrioid, endometrial carcinomas, and their precursor lesions. Squamous, secretory, or mucinous metaplasia are common, and when seen present both diagnostic and conceptual challenges. A central problem is that a change in differentiation state can be seen in either benign or neoplastic endometrium. For this reason, endometrial 'metaplasias' are best thought of simply as an altered differentiation state, in which the context, molecular biology, and subsequent natural history codetermine whether an individual example is benign, premalignant, or malignant.

Squamous differentiation within type I endometrial glandular lesions occurs in approximately $25 \%$ of adenocarcinomas, ${ }^{3}$ and in many premalignant $\mathrm{EIN}^{4,5}$ lesions. Although it is a common finding that precancerous lesions display focal squamous differentiation in the form of expansile intraglandular morules, the resultant carcinomas are almost always of glandular (rather than squamous) morphology. This has suggested the possibility that the risk for subsequent endometrial carcinoma in women with endometrial squamous morules might be primarily dependent on the presence or absence of a coexisting glandular atypical hyperplasia or EIN lesion. In this study, we test that hypothesis by correlating the histologic appearance of morule-associated endometrial glands with clinical progression to adenocarcinoma. Then, with a subset of cases, we examine clonal relationships and hormonal competence of admixed squamous and glandular elements to understand how these arise and behave in response to the hormonal environment.

\section{Materials and methods}

\section{Case Selection}

Initial ('Index') morule-bearing endometrial biopsies were obtained by pathology report review of the Department of Pathology at Brigham and Women's Hospital for the interval 1989-1999. Hematoxylin and eosin (H\&E)-stained sections from each specimen were reviewed by three pathologists (M-CL, TAI, and GLM) and a consensus diagnosis obtained. Cases of atypical polypoid adenomyoma and those without H\&E slides were excluded. H\&E slides from each sample were reviewed; morular lesions in the index specimens were classified according to the degree of coexisting glandular complexity as follows: class A, isolated morules or morules unassociated with a glandular lesion; class B, morules associated with a localizing glandular lesion sub- diagnostic of EIN; class C, morules associated with EIN. All premalignant lesions studied met diagnostic criteria for both $\mathrm{EIN}^{2,4}$ and atypical endometrial hyperplasia. Although in general these diagnoses do not always exactly correlate, ${ }^{6}$ their convergence in the specific cases within this series make them interchangeable here for purposes of discussion. Follow up was conducted via survey of the pathology record, and review of histologic outcomes. Outcomes were classified as regression (no morules or glandular lesion), persistence (morules or EIN retained), or progression to adenocarcinoma.

A total of eight curettage cases diagnosed as EIN with squamous morules underwent immunohistochemical and genomic analysis as described below.

The project was reviewed and approved by the institutional review board for the Protection of Human Subjects of Brigham and Women's Hospital. None of the authors have financial or personal conflicts with the material included in this report. This work was presented in part at the 2005 annual meeting of the American Association for Cancer Research.

\section{Immunohistochemical Analysis}

One paraffin block containing representative areas of the lesion was selected from each case. We evaluated the estrogen receptor (ER) and progesterone receptor (PR) status of neoplastic glands and squamous areas, respectively. In addition, we compared the cellular proliferation status between glandular and squamous elements by immunohistochemical assessment of the percentage of nuclei that express the proliferative marker Ki-67 antigen. Immunohistochemistry was performed essentially as described previously. ${ }^{7}$ Briefly, deparaffinized rehydrated $5-\mu \mathrm{m}$ paraffin sections underwent microwave antigen retrieval before adding primary antibody. Mouse anti-human anti-ER antibody (ID5; Dako, Carpinteria, CA, USA), mouse anti-human anti-PR antibody (IA6; Dako), mouse anti-human anti-Ki-67 antigen antibody (MIB-1; Dako), and murine anti-human PTEN antibody (6h2.1; Cascade Biosciences, Winchester, MA, USA) were used at $1: 300,1: 100,1: 100$, and $1: 100$ dilution, respectively. Primary antibody was incubated overnight at $4^{\circ} \mathrm{C}$, washed, incubated with appropriate secondary biotinylated immunoglobulin (Vectastain ABC kit; Vector Laboratories Inc., Burlingame, CA, USA) and signal detected by sequential addition of avidin peroxidase and 3,3'-diaminobenzidine. The staining was scored by consensus of three pathologists (M-CL, TAI, and GLM). Endometrial stroma and/or normal endometrial epithelium provided an internal positive control, and negative control without addition of primary antibody showed low background staining in all cases.

Specimens were considered hormone receptor positive when nuclear staining was visible in 
a minimum of $20 \%$ of cells. Ki-67 index was expressed as the fraction of MIB-1 positively labeled epithelial cells (\% positive). Positively labeled cells were defined as any nucleus with detectable staining compared with the negative control background level. PTEN status was scored visually as negative (-, PTEN null), if lesional endometrial glands showed no PTEN immunohistochemical signal relative to the background positive endometrial stroma, and positive ( + , PTEN expressing), if endometrial glands showed nuclear and cytoplasmic PTEN protein.

\section{Genomic Analysis}

PTEN mutational analysis was undertaken on one EIN case demonstrated to be null for the PTEN protein by immunohistochemistry. Glandular and squamous elements of affected areas were separately isolated from paraffin sections using laser capture microdissection (PixCell LCM System; Arcturus Bioscience Inc., Mountain View, CA, USA). Purified DNA from each component was PCR amplified using primers which define the coding region and flanking introns of all nine PTEN gene exons and mutations identified using previously described primers and protocols. ${ }^{8}$ In summary, PCR products were subjected to denaturing gradient gel electrophoresis and DNA samples showing electrophoretic migration variants underwent reamplification and semiautomated direct sequencing (Model 3700 DNA sequencer; Applied Biosystems, Foster City, CA, USA).

\section{Results}

\section{Clinical Outcomes of Cases Studied}

In total, 127 cases were retrieved; 6 cases of atypical polypoid adenomyoma, 2 cases with concurrent carcinoma at initial diagnosis, and 14 cases with no available H\&E slides were excluded from analysis. Of the 105 remaining cases, mean age was 53.9 years, median 53 years. The distribution of glandular patterns in the index biopsy of 105 accepted cases was 50\% (52/105) class A, 18\% (19/105) class B, and $32 \%(34 / 105)$ class C. Figure 1 shows examples of the histologic appearances of these classes of morule bearing lesions. Of 66 cases with clinical follow-up, patients had 1-12 subsequent biopsies (average $=2.5$, median $=2$, total 168), over a follow-up interval from 1 to 146 months (average $=31.2$, median $=12.5$ ). Clinical outcome was significantly (Table $1, \chi^{2}$-test, $P=0.049$ ) associated with the glandular pattern seen in the morulebearing index biopsy. A total of $11 \%$ (7/66) of patients were found to have carcinoma on follow up, of which most $(71 \%, 5 / 7)$ had a glandular EIN lesion in the index biopsy. The interval from index biopsy to carcinoma averaged 2.9 years overall (median 2), but was longer for class A (average 8.2 years) biopsies compared with class C (0.8 years).

\section{Immunohistochemical Analysis}

The results of immunostaining are summarized in Table 2 .

In all cases, the EIN glandular components had abundant nuclear localization of ER and PR. In
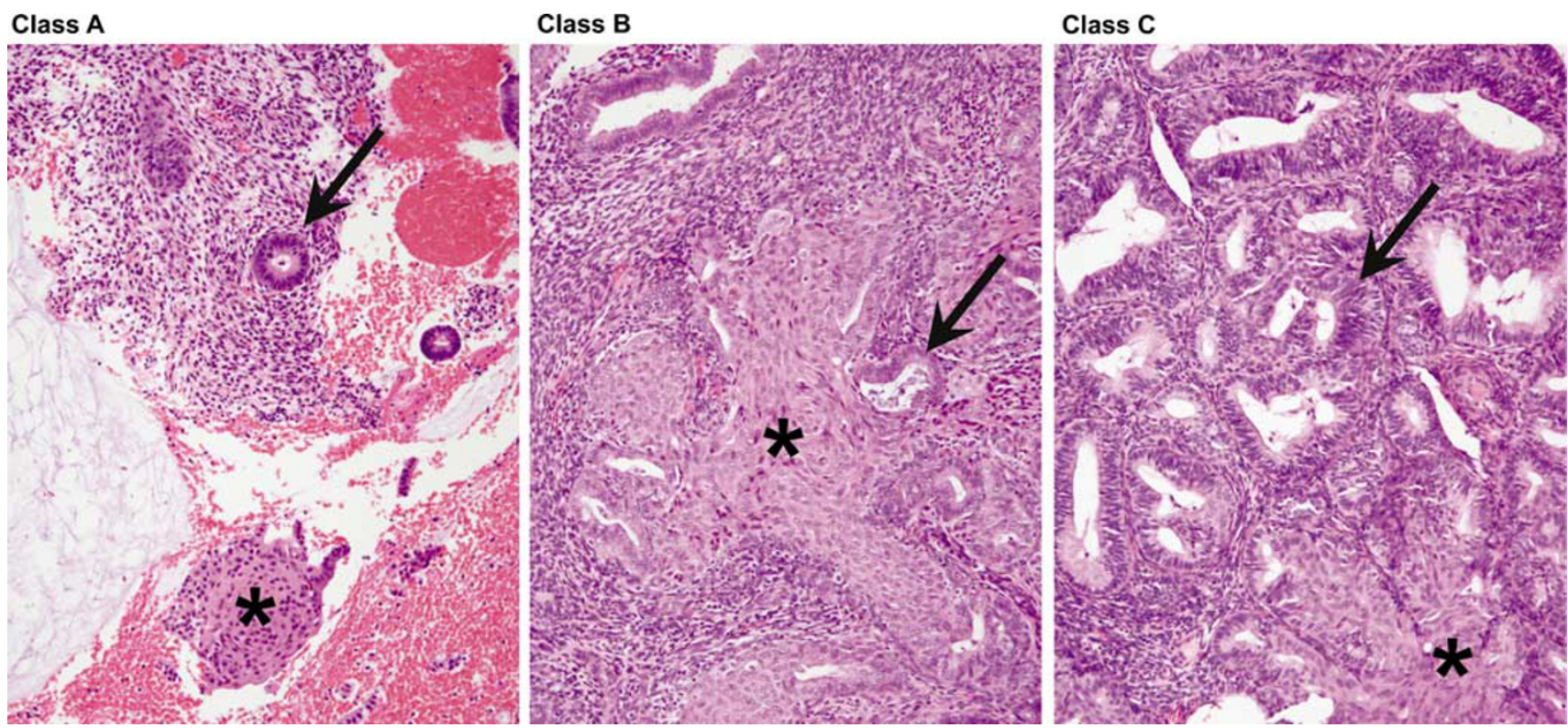

Figure 1 Glandular patterns in endometria with squamous morules. Squamous morules (asterisk) may be seen in a variety of glandular contexts (arrow) including: class A (left), normal appearing glands; class B (center) localized glandular lesion lacking sufficient density or cytologic changes for EIN; or class C (right), premalignant EIN lesions having altered gland cytology and gland area in excess of stromal area 
Table 1 Clinical outcomes by initial glandular pattern

\begin{tabular}{llllll}
\hline Intake DX & $\begin{array}{c}\text { Total } \% \\
(\mathrm{n})\end{array}$ & $\begin{array}{c}\text { Regression } \\
\%(\mathrm{n})\end{array}$ & $\begin{array}{c}\text { Persistence } \\
\%(\mathrm{n})\end{array}$ & $\begin{array}{c}\text { Cancer \% } \\
\text { (n) }\end{array}$ & $\begin{array}{c}\text { Interval to cancer, } \\
\text { mean months (range) }\end{array}$ \\
\hline $\begin{array}{l}\text { Class A morule: Isolated morules without glandular lesion } \\
\text { Class B morule: Morules with focal gland crowding but } \\
\text { subdiagnostic of EIN }\end{array}$ & $\begin{array}{l}100(31) \\
100(9)\end{array}$ & $\begin{array}{c}77.4(24) \\
88.9(8)\end{array}$ & $\begin{array}{c}16.1(5) \\
11.1(1)\end{array}$ & $\begin{array}{c}6.5(2) \\
0(0)\end{array}$ & $98(50-146)$ \\
Class C morule: Morules with EIN & $100(26)$ & $42.3(11)$ & $38.5(10)$ & $19.2(5)$ & $10(1-45)$ \\
Total & $100(66)$ & $65.2(43)$ & $24.2(16)$ & $10.6(7)$ & $35(1-146)$ \\
\hline
\end{tabular}

EIN, endometrial intraepithelial neoplasia. $\chi^{2}, P=0.049$.

Table 2 Estrogen receptor (ER) and progesterone receptor (PR) and Ki-67 proliferation indices between glandular and squamous components of endometrial neoplasia

\begin{tabular}{|c|c|c|c|c|c|c|c|c|}
\hline \multirow[t]{2}{*}{ Case } & \multirow[t]{2}{*}{ Diagnosis $^{\mathrm{a}}$} & \multirow[t]{2}{*}{$P T E N^{\mathrm{b}}$} & \multicolumn{3}{|c|}{ Glandular components ${ }^{\mathrm{a}}$} & \multicolumn{3}{|c|}{ Squamous component ${ }^{\mathrm{a}}$} \\
\hline & & & $E R^{\mathrm{b}}$ & $P R^{\mathrm{b}}$ & Ki-67 $(\%)^{\mathrm{c}}$ & $E R^{\mathrm{b}}$ & $P R^{\mathrm{b}}$ & Ki-67 (\%) \\
\hline 1 & EIN, morules & + & + & + & 15 & - & - & 0 \\
\hline 2 & EIN, morules & + & + & + & 5 & - & - & 0 \\
\hline 3 & EIN, morules & + & + & + & 5 & - & - & 0 \\
\hline 4 & EIN, morules & + & + & + & 15 & - & - & 0 \\
\hline 5 & EIN, morules & + & + & + & 15 & - & - & 1 \\
\hline 6 & EIN, morules & + & + & + & 10 & - & - & 0 \\
\hline 7 & EIN, morules & + & + & + & 2 & - & - & 0 \\
\hline 8 & EIN, morules & - & + & + & 10 & - & - & 1 \\
\hline
\end{tabular}

EIN, endometrial intraepithelial neoplasia; ER, Estrogen receptor; PR, progesterone receptor; PTEN, phosphatase and tensin homolog deleted on chromosome 10.

aPremalignant (EIN) endometrial glandular lesions with expansile luminal squamous morules ('morules') or uterine surface lining squamous differentiation ('squamous') were subjected to immunohistochemical analysis as described in 'Materials and methods'.

${ }^{\mathrm{b}}$ For ER, PR, and PTEN staining is recorded as present (+) or absent (-).

${ }^{\mathrm{C}}$ Proliferative (Ki-67) index measured as percentage of cells with nuclear staining.

contrast, the squamous components were always negative for ER and PR (Figure 2c and d).

With respect to cellular proliferation status of glandular components of EIN lesions (Figure 2b), Ki-67-staining extent ranged from 2 to $15 \%$. In contrast, the squamous components had absent or extremely low levels ( $<1 \%$ of cells) of Ki-67 staining (Table 2). PTEN protein was expressed in both lesional glands and squamous elements in $88 \%$ (7/8) of EIN lesions (Table 2).

\section{Genomic PTEN Analysis}

The EIN case that was immunohistochemically null for PTEN protein was confirmed to contain a mutation (exon 1,7 9delGCC, insTT) of the PTEN gene (Figure $3 \mathrm{~b}$ ) which was identically conserved in separate samples of neoplastic EIN glands and squamous morules, but absent in control normal glands and endometrial stroma (Figure 3a). This confirms a common clonal composition between glandular and squamous EIN elements, and somatic, rather than heritable, mutation as the mechanism of genomic PTEN inactivation.

\section{Discussion}

The glands of squamous morule-bearing endometrial lesions demonstrate a variety of appearances, most commonly $(50 \%)$ of normal density and cytology, but less frequently with mild gland crowding $(18 \%)$ or EIN (32\%). These histologic types are associated with differing clinical outcomes (Table 1), with most of the cases that progressed to cancer $(5 / 7)$ being preceded by morules located within EIN glandular lesions. Progression interval was quite short for EIN lesions (average 0.8 years) compared with that for isolated squamous morules (two cases developed carcinoma after 4 and 14.6 years). The likelihood of progression of morulebearing EIN lesions to carcinoma $(19 \%$ in this series), although significantly less than the $41 \%$ seen previously for all types of EIN ${ }^{9}$ justifies therapeutic intervention. For those patients with isolated squamous morules but no EIN, reevaluation or close surveillance will eventually uncover carcinoma in a fraction $(6.5 \%)$ of patients.

Conservation of identical mutations in admixed squamous and glandular elements of premalignant (EIN, atypical hyperplasia) and malignant (cancer) 
a

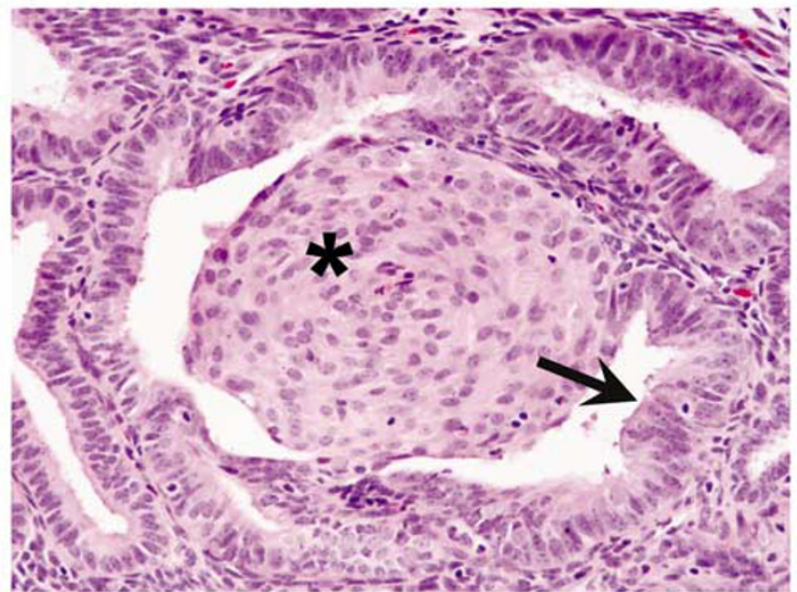

C

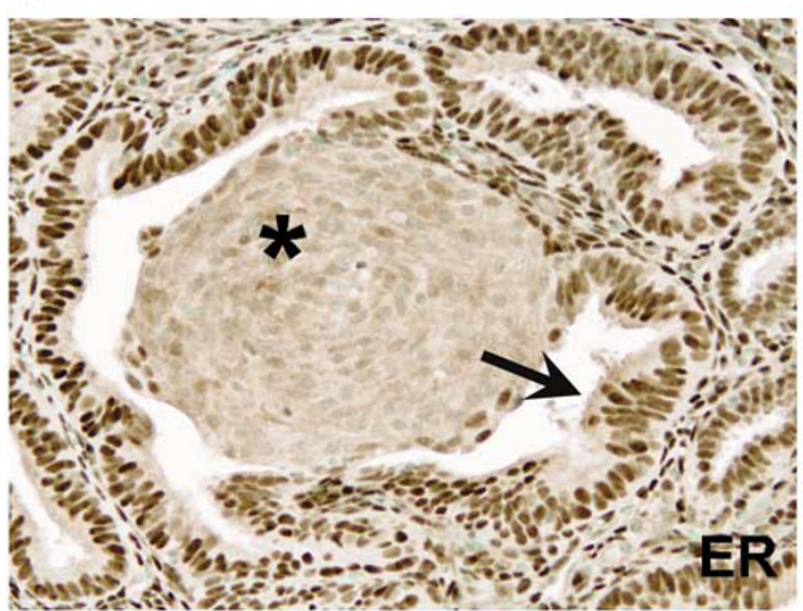

b

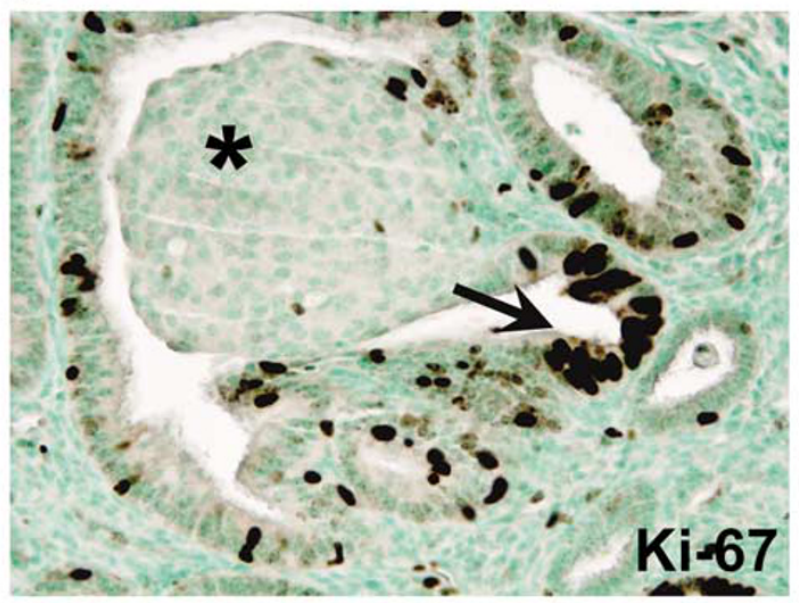

d

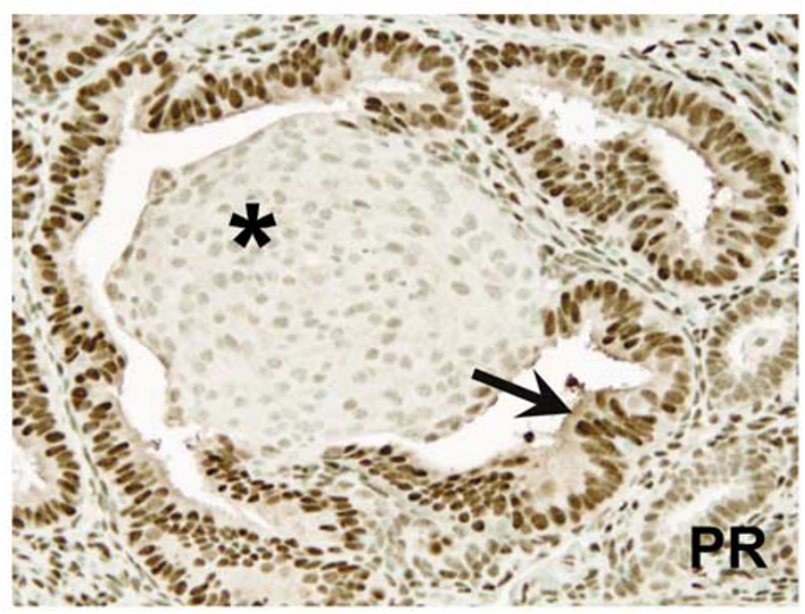

Figure 2 Loss of hormonal competence in squamous morules but not glands. Immunohistochemical expression of estrogen receptor (ER, c), progesterone receptor (PR, d), and mitotic activity (Ki-67, b) in EIN with squamous morules (case 8) shows squamous morules (asterisk) are essentially negative for ER, PR, and Ki-67, in contrast with glandular (arrow) components; (a) hematoxylin and eosin stain.
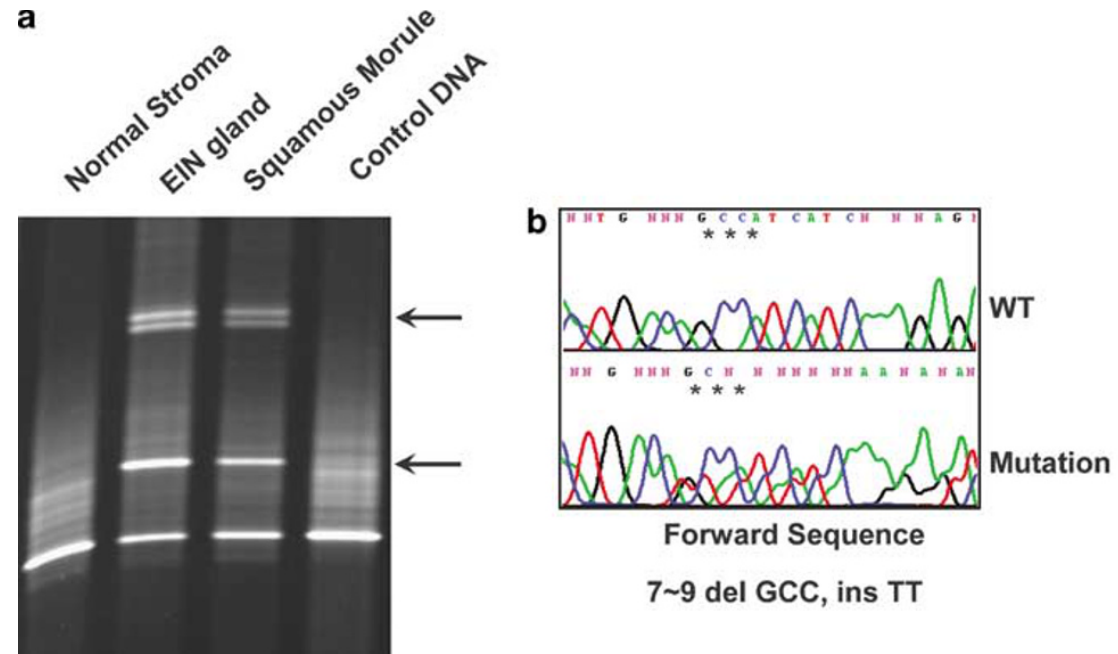

Figure 3 Squamous and glandular components from a common premalignant lineage undergo divergent differentiation. Identical PTEN mutation in the matched squamous and glandular elements of an EIN lesion was confirmed by immunodirected laser capture microdissection of DNA. (a) PTEN-null glands and squamous morules of EIN have matched aberrantly migrating bands in a screening denaturing gradient electrophoresis gel (arrowheads) compared with PTEN-expressing endometrial stroma. (b) Direct sequencing confirms identical Exon 1 mutation $7 \sim 9$ del GCC, insTT, in squamous and glandular components. 
endometrial neoplasia of individual patients confirms the hypothesis that both squamous and glandular elements are integral components of a common progenitor cell. Squamous elements, therefore, are not a reactive process involving nonneoplastic tissues. Somatic PTEN mutations are sufficiently unique as to be good lineage-specific markers. Because each occurrence of a new PTEN mutation is widely scattered across all nine exons of the approximately $3 \mathrm{~kb}$ gene, ${ }^{8,10-12}$ they generally differ between patients and among independent multicentric events occurring within the same patient. Additional evidence for a shared lineage is supplied by other published studies showing conservation of the same inactive X chromosome copy, ${ }^{13}$ and particular $\beta$-catenin mutations, ${ }^{14}$ between squamous and glandular components of neoplastic endometrial lesions.

Acquired hormonal incompetence and amitotic state in the squamous but not glandular component may be a manifestation of terminal cellular commitment, making the functionally inert squamous subpopulation an unlikely participant in estrogendriven lesion progression from premalignant to malignant disease. This is the basis for the recommendation that gland-crowding assessments used to diagnose EIN (area of glands must exceed that of stroma) must be performed to the exclusion of squamous components. ${ }^{2}$ Malignancies which follow morule-containing EIN lesions are usually pure adenocarcinomas rather than squamous carcinomas. ${ }^{15}$ Other investigators have previously shown coordinate loss of ER and PR, and reduction of Ki-67 staining specifically in the squamous but not glandular elements of endometrial adenocarcinomas. ${ }^{16,17}$ Our work extends this observation to premalignant EIN lesions. Similarly, BCL2 expres- sion in endometrial epithelial tissues requires an intact progesterone response pathway, ${ }^{18}$ so loss of $\mathrm{PR}$ in morule components is a likely contributor to the lower levels of BCL2 expression seen in squamous morules. ${ }^{19}$

The combination of high somatic activation rates in sporadic squamous-containing lesions, known transforming activities, and experimental association with induction of a squamous phenotype ${ }^{20}$ have now established mutation of the gene encoding $\beta$-catenin, ${ }^{20}$ rather than PTEN, as the most frequent event seen in squamous-containing endometrial glandular premalignant lesions. The frequency of PTEN inactivation in squamous morule-containing EIN lesions $(13 \%, 1 / 8)$ is significantly lower compared with the $63 \%(22 / 35)$ inactivation reported previously for all EIN lesions. ${ }^{7}$ The gene encoding $\beta$-catenin is mutated in up to $86 \%$ of endometrial carcinomas with squamous differentiation, in contrast to $13 \%$ lacking a squamous component. ${ }^{14}$ Similarly, $\beta$-catenin gene mutations are frequent in premalignant endometrial lesions with squamous morules $(54 \%)$ but rare $(9 \%)$ in those without. ${ }^{21}$

Progestin therapy has been long known to be effective in the treatment of precancerous, and even malignant, glandular endometrial lesions. ${ }^{22-25}$ For those EIN lesions with squamous morules, we would predict morules to be relatively resistant to the apoptotic effects of progestins, ${ }^{26}$ disappearing only when shedding of the adjacent endometrium carries them away with a withdrawal bleed. Indication of differentiation-state-specific response to progestins is provided by comparison of squamous and glandular EIN histopathology before and after high-dose progestin therapy. Figure 4 shows a morule-bearing EIN lesion which after several months of progestin therapy responds by developing a

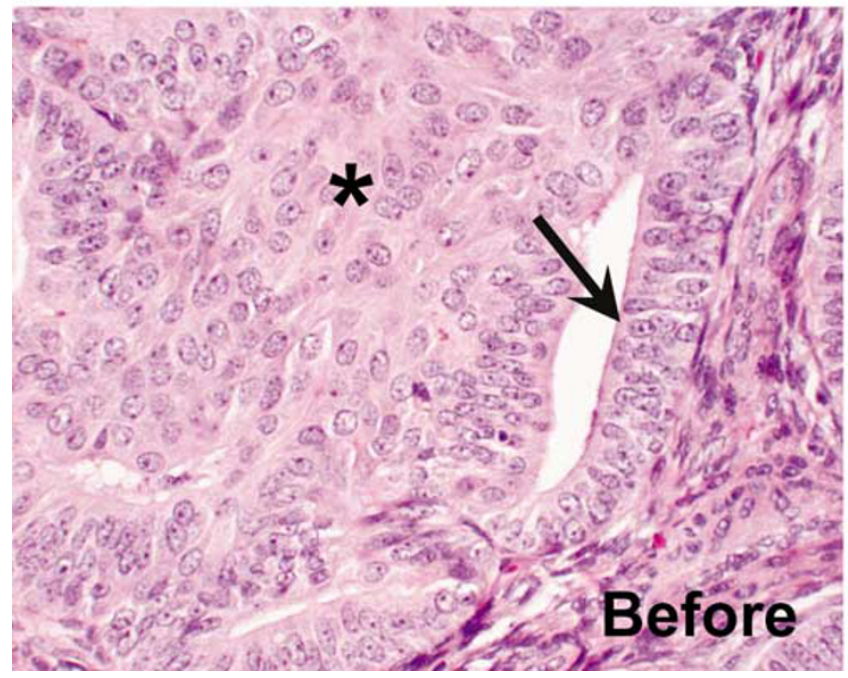

b

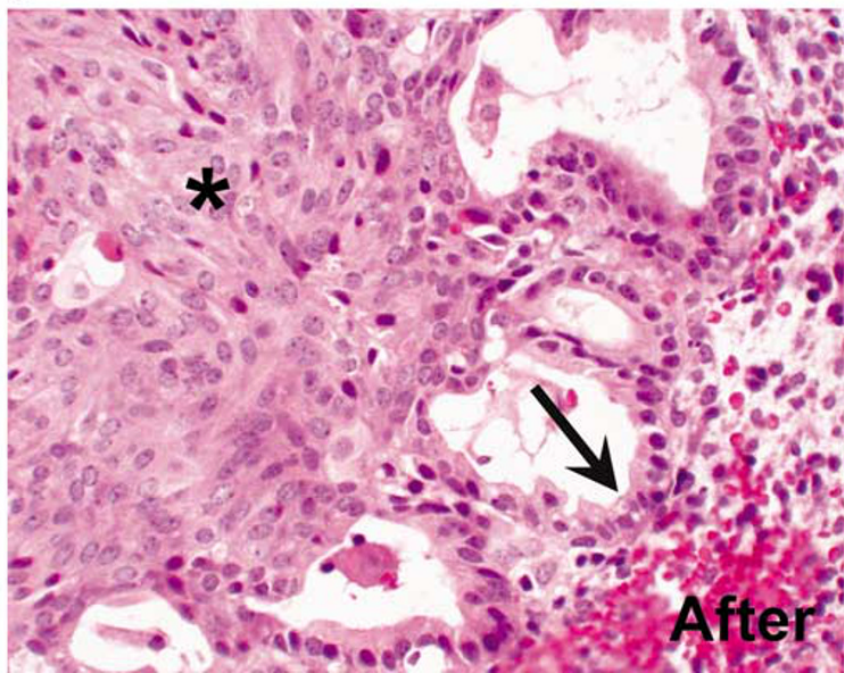

Figure 4 Selective effects of progestin therapy on glandular and squamous elements of EIN. A 42-year-old woman with EIN showing squamous morules was treated with 4 months of high-dose progestins. After therapy, the squamous components are unchanged (asterisk), but cells of the glandular epithelium (arrows) demonstrate reduction of epithelial thickness and nuclear size. 
degenerative nuclear pyknosis and cytoplasmic shrinkage of the glandular, but not squamous, components.

We can now summarize a model of endometrial morule-containing precancerous lesions consistent with the evidence presented above. Intraglandular morules arise as a transdifferentiation event from a preexisting clone in which the glandular and squamous elements contain conserved $\beta$-catenin mutations. The frequency and distribution of squamous morules within preexisting glandular lesions is more consistent with a tendency for squamous differentiation within the neoplastic field, rather than independent new mutational events within each morule. Only the glandular elements retain competence to respond to the cancer-promoting effects of estrogens, or involuting effects of progestins. Cancers which result are thus derived from the glandular elements, and primarily have a glandular phenotype. If the lesion is treated with progestins, or exposed to progestins during natural menstrual cycles, the glandular components are prone to involution, thereby proportionately enriching for the squamous elements. A diagnostic problem presents itself when only isolated squamous morules are found in an endometrial biopsy, as it is not always possible to tell whether it represents a successfully treated glandular lesion with residua of hormonally inert morules, or an incompletely sampled neoplastic process. Both are possible mechanisms for presentation of isolated squamous morules in an otherwise unremarkable endometrial biopsy.

The diagnostic classification of squamous morulebearing endometrial biopsies is primarily accomplished based on the histologic appearance of the glandular component. In cancer-free patients with endometrial squamous morules, prospective endometrial cancer risk is primarily determined by presence or absence of an EIN glandular lesion, in keeping with the notion that it is the glandular rather than squamous components, which are susceptible to the cancer risk modulating effects of estrogens and progestins. The finding of isolated squamous morules without any evident glandular abnormalities, however, justifies resampling and careful follow up.

\section{Acknowledgements}

We thank Xiao-ping Zhou (Genomic Medicine Institute, Cleveland Clinic Foundation, Cleveland, $\mathrm{OH}$, and Human Cancer Genetics Program, The Ohio State University, Columbus, $\mathrm{OH}$ ) for technical assistance in PTEN mutational analysis. This work was supported by NIH grants RO1-CA100833 and R01-CA92301 (G Mutter), NHRI Physician Scientist Award, Taiwan (M Lin) and the Helse Vest, Norway (JB). CE is a recipient of the Doris Duke Distinguished Clinical Scientist Award.

\section{Disclosure/conflict of interest}

All the authors declared no conflict of interests.

\section{References}

1 Bokhman J. Two pathogenetic types of endometrial carcinoma. Gynecol Oncol 1983;15:10-17.

2 Mutter GL, Zaino RJ, Baak JPA, et al. The benign endometrial hyperplasia sequence and endometrial intraepithelial neoplasia. Int J Gynecol Pathol 2007;26:103-114.

3 Zaino RJ, Kurman RJ. Squamous differentiation in carcinoma of the endometrium: a critical appraisal of adenoacanthoma and adenosquamous carcinoma. Semin Diagn Pathol 1988;5:154-171.

4 Silverberg SG, Mutter GL, Kurman RJ, et al. Tumors of the uterine corpus: epithelial tumors and related lesions. In: Tavassoli FA, Stratton MR (eds). WHO Classification of Tumors: Pathology and Genetics of Tumors of the Breast and Female Genital Organs, 1st edn. IARC Press: Lyon, France, 2003, pp 221-232.

5 Mutter GL. Diagnosis of premalignant endometrial disease. J Clin Pathol 2002;55:326-331.

6 Hecht JL, Ince TA, Baak JP, et al. Prediction of endometrial carcinoma by subjective endometrial intraepithelial neoplasia diagnosis. Mod Pathol 2005;18:324-330.

7 Mutter GL, Ince TA, Baak JPA, et al. Molecular identification of latent precancers in histologically normal endometrium. Cancer Res 2001;61:4311-4314.

8 Mutter GL, Lin MC, Fitzgerald JT, et al. Altered PTEN expression as a diagnostic marker for the earliest endometrial precancers. J Natl Cancer Inst 2000;92:924-930.

9 Baak JP, Mutter GL, Robboy S, et al. The molecular genetics and morphometry-based endometrial intraepithelial neoplasia classification system predicts disease progression in endometrial hyperplasia more accurately than the 1994 World Health Organization classification system. Cancer 2005;103:2304-2312.

10 Risinger JI, Hayes AK, Berchuck A, et al. PTEN/ MMAC1 mutations in endometrial cancers. Cancer Res 1997;57:4736-4738.

11 Tashiro H, Blazes MS, Wu R, et al. Mutations in PTEN are frequent in endometrial carcinoma but rare in other common gynecological malignancies. Cancer Res 1997;57:3935-3940.

12 Maxwell G, Risinger J, Gumbs C, et al. Mutation of the PTEN tumor supressor gene in endometrial hyperplasias. Cancer Res 1998;58:2500-2503.

13 Mutter GL, Baak JPA, Crum CP, et al. Endometrial precancer diagnosis by histopathology, clonal analysis, and computerized morphometry. J Pathol 2000;190:462-469.

14 Saegusa M, Okayasu I. Frequent nuclear beta-catenin accumulation and associated mutations in endometrioid-type endometrial and ovarian carcinomas with squamous differentiation. J Pathol 2001;194:59-67.

15 Yamashina M, Kobara TY. Primary squamous cell carcinoma with its spindle cell variant in the endometrium. A case report and review of literature. Cancer 1986;57:340-345.

16 Lax SF, Pizer ES, Ronnett BM, et al. Comparison of estrogen and progesterone receptor, Ki-67, and p53 
immunoreactivity in uterine endometrioid carcinoma and endometrioid carcinoma with squamous, mucinous, secretory, and ciliated cell differentiation. Hum Pathol 1998;29:924-931.

17 Nielsen AL, Nyholm HC. Endometrial adenocarcinoma of endometrioid subtype with squamous differentiation: an immunohistochemical study of MIB-1 (ki-67 paraffin), cathepsin D, and C-erbB-2 protein (p185). Am J Pathol 1995;14:230-234.

18 Saegusa M, Kamata Y, Isono M, et al. Bcl-2 expression is correlated with a low apoptotic index and associated with progesterone receptor immunoreactivity in endometrial carcinomas. J Pathol 1996;180:275-282.

19 Saegusa M, Okayasu I. Down-regulation of bcl-2 expression is closely related to squamous differentiation and progesterone therapy in endometrial carcinomas. J Pathol 1997;182:429-436.

20 Miyoshi K, Hennighausen L. Beta-catenin: a transforming actor on many stages. Breast Cancer Res 2003;5:63-68.

21 Brachtel EF, Sanchez-Estevez C, Moreno-Bueno G, et al. Distinct molecular alterations in complex endometrial hyperplasia (CEH) with and without immature squamous metaplasia (squamous morules). Am J Surg Pathol 2005;29:1322-1329.

22 Kim YB, Holschneider CH, Ghosh K, et al. Progestin alone as primary treatment of endometrial carcinoma in premenopausal women. Report of seven cases and review of the literature. Cancer 1997;79: 320-327.

23 Zheng W, Baker HE, Mutter GL. Involution of PTENnull endometrial glands with progestin therapy. Gynecol Oncol 2004;92:1008-1013.

24 Montz FJ, Bristow RE, Bovicelli A, et al. Intrauterine progesterone treatment of early endometrial cancer. Am J Obstet Gynecol 2002;186:651-657.

25 Wang S, Pudney J, Song J, et al. Mechanisms involved in the evolution of progestin resistence in human endometrial hyperplasia-precursor of endometrial cancer. Gynecol Oncol 2003;88:108-117.

26 Amezcua CA, Lu JJ, Felix JC, et al. Apoptosis may be an early event of progestin therapy for endometrial hyperplasia. Gynecol Oncol 2000;79:169-176. 